\title{
Reliability and validity of the Strengths and Difficulties Questionnaire in Greek adolescents and their parents
}

\author{
George Giannakopoulos ${ }^{1,2^{\star}}$, Christine Dimitrakaki ${ }^{1}$, Kalliroi Papadopoulou $^{3}$, Chara Tzavara $^{1}$, \\ Gerasimos Kolaitis ${ }^{2}$, Ulricke Ravens-Sieberer ${ }^{4}$, Yannis Tountas ${ }^{1}$ \\ ${ }^{1}$ Centre for Health Services Research, Department of Hygiene, Epidemiology and Medical Statistics, Athens University Medical School, \\ Athens, Greece; ${ }^{*}$ Corresponding Author: giannakopoulos.med@gmail.com \\ ${ }^{2}$ Department of Child Psychiatry, Athens University Medical School, Aghia Sophia Children's Hospital, Athens, Greece \\ ${ }^{3}$ Faculty of Early Childhood Education, Athens University School of Education, Athens, Greece \\ ${ }^{4}$ Robert Koch Institute, Child and Adolescent Health, Berlin, Germany
}

Received 30 August 2013; revised 30 September 2013; accepted 20 October 2013

Copyright (C) 2013 George Giannakopoulos et al. This is an open access article distributed under the Creative Commons Attribution License, which permits unrestricted use, distribution, and reproduction in any medium, provided the original work is properly cited.

\section{ABSTRACT}

The Strengths and Difficulties Questionnaire (SDQ) is a brief screening measure of emotional and behavioural difficulties in children and adolescents. The aim of this study was to assess reliability and validity of the Greek version of the SDQ. A representative Greek sample of 1194 adolescents (aged 11 to 17 years) and their parents completed the SDQ along with other measures. Internal consistency reliability was determined by calculation of the Cronbach $a$ coefficient. Varimax Orthogonal Transformation was conducted to test the factor structure of the questionnaire. Validity was further examined by investigating the correlation of the SDQ with the KIDSCREEN questionnaire and its association with demographic factors. The inter-rater agreement between parent and self-reports was analyzed with Pearson's correlation coefficient. Intra-class correlation coefficients (ICCs) were computed to determine test-retest stability. For both parent and the self-report SDQ versions, most items loaded onto their predicted factors in consistency with the originally proposed five-factor structure. Internal consistency reliability was acceptable with a Cronbach $\alpha$ above 0.70 for all SDQ scales except for conduct and peer problems. Inter-rater correlations ranged from 0.33 to 0.45 . Test-retest stability was good (ICCs $>0.60$ ). Correlation coefficients between the SDQ and KIDSCREEN questionnaire were significant. Small effect sizes $(d>0.5)$ of the socioeconomic status were found for all of the SDQ scale mean scores. In conclusion, the SDQ was found to have satisfying psychometric properties and could be suitable for assessing emotional and behavioural problems in Greek adolescents.

Keywords: Adolescents; Emotional and Behavioural Problems; Strengths and Difficulties Questionnaire; Reliability; Validity

\section{INTRODUCTION}

Validated questionnaires for identifying emotional and behavioural problems and measuring psychopathological symptoms in children and adolescents in community, school and clinical settings are of considerable value. The reason for this is twofold. Firstly, only a few children and adolescents eventually make use of mental health services, although the prevalence rates of emotional and behavioural problems are high internationally. It is notable that levels rise dramatically (for example up to $40 \%$ ), particularly in disadvantaged areas, when more general difficulties and not specific psychiatric disorders are concerned $[1,2]$. Therefore, questionnaires with the potential to accurately detect children at risk for developing emotional and behavioural problems are of crucial importance. Secondly, health professionals can use such measures in their practice so as to assess the type, the severity and the impact of the problem, as well as to evaluate the effects of a treatment or preventive intervention [3].

The Strengths and Difficulties Questionnaire (SDQ) is a brief screening instrument developed for the above- 
mentioned purposes [4]. It has been translated into more than 40 languages in recent years, meeting the need for a practical, economic and user-friendly instrument [5]. Versions are available for self-reporting by adolescents aged 11 to 16 years, as well as for parents and teachers of children and adolescents aged 4 to 16 years. The SDQ is advantageous compared to related instruments in that it is very short and includes both strengths and difficulties. The psychometric properties of the SDQ have been investigated in numerous studies with community samples in different countries [6-36]. All these studies have consistently shown that the factor analysis of SDQ parent, teacher and/or self-report versions suggests a five-factor structure, i.e., emotional symptoms, conduct problems, hyperactivity/inattention, peer problems and prosocial behaviour scale, that corresponds with the domains of psychopathology and personal strengths it intends to measure.

The reliability of the SDQ can be defined as satisfactory, although it should be noted that the internal consistency of the conduct problems scale in parent and selfreports as well as the peer problems scale in the selfreported version are low, probably due to the limited number of items [10,14,24,27,31,34,37,38]. Test-retest stability of the SDQ is also satisfactory [6,18,25,26,28, $33,34]$, although a study found that test-retest stability was generally lower than it would be expected [38]. Correlations among parent, teacher and self-reported SDQ scores are found to be moderate, yet better than cross-informant correlations of other psychopathology measures [6,26,38-40]. Finally, much evidence has supported the concurrent validity of the SDQ in conjunction with other related established measures as well as its discriminant validity with self-reports discriminating more on ratings of emotional and peer problems, and parent/teacher reports discriminating more on hyperactivity symptoms [25,27,29,32,33,39,41].

The present study aimed at assessing the psychometric properties of the self-reported and parent-reported SDQ in Greece. A representative nation-wide sample of adolescents and their parents completed the questionnaire, along with a number of related measures in order to examine validity issues. The following issues were investigated: 1) the factor structure of the Greek SDQ, 2) its internal consistency; 3) inter-rater and test-retest agreement; 4) the gender and age effects and 5) validity issues such as construct and convergent validity.

\section{METHODS}

\subsection{Participants and Procedure}

This study was conducted within the framework of the European project "Screening and Promotion for HealthRelated Quality of Life (HRQoL) in Children and Ado- lescents: A European Public Health Perspective" [42]. The school sampling in Greece was random, multistaged and performed to take into account distribution of the target population by age and administrative school region. The target population was adolescents aged 11 to 17 years. A sample size of 1800 adolescents was considered necessary to detect a minimally important difference of half a standard deviation (SD) in HRQoL scores within each age strata between children with and without special healthcare needs or a chronic condition. A response rate of approximately $70 \%$ was expected, so the initial sample size was set at 2400 children and adolescents. In Greece, ages 11 to 17 years correspond to six secondary school grades.

Approximately 400 students were included from each of the six age groups/grades in order to reach the original target of 2400 adolescents. For example, the total number of students in Greece attending the first grade of the secondary school is 119.055 . If an administrative region had a total number of 2174 students attending the first grade of the secondary school, then 8 students were randomly recruited from a school in that region $((2174 \times$ $400) / 119,055=7.6$ students). Each age group/grade had been calculating accordingly, for each sector. Schools in each sector were randomly selected by a computer program and students of each selected school were selected randomly from classroom name lists. A sample of 1900 adolescents (aged 11 to 17 years) and their parents was recruited. A total of 1194 (that is, a 63\% response rate) of self-reported (479 boys and 715 girls) and 1194 respective parent-reported questionnaires were returned. Total adolescent sample mean age was 13.6 years $(\mathrm{SD}=$ 1.7). Regarding the socioeconomic status (SES) characteristics of the sample, $37.59 \%$ came from low-income families, $44.96 \%$ came from middle-income families and $17.45 \%$ from high-income families. Students and parents were asked to complete the questionnaire at home after providing written informed consent. The SDQ was readministered two to three weeks later to a randomly selected $10 \%$ of the sample. Inclusion criteria were adequate reading skills. Previous research on the representativeness of the present sample has reported that non-responder interviews showed no significant differences between responders and non-responders with regard to adolescents' and parents' general perceived health, parents' marital status and highest educational level, and type of residence, indicating that a selection bias is less likely [42].

\subsection{Measures}

The SDQ contains 25 items (small sentences), categorised into 5 scales of 5 items each: hyperactivity/inattention, emotional symptoms, conduct problems, peer problems and prosocial behaviour [4]. Responses to each 
of the 25 items consisted of 3 options: not true, somewhat true, or certainly true. For all scales the items that are worded negatively are assigned scores of 2 for certainly true, 1 for somewhat true, and 0 for not true. Versions for self-report and parent-report were used in the present study. In order to combat inherent weaknesses of cross-cultural adaptation (for example, semantic and scale equivalence) the research team in the present study followed a standardised translation methodology according to international cross-cultural translation guidelines [43].

To assess family income, the Family Affluence Scale (FAS) [44] was used, addressing issues of family car ownership, having their own unshared room, the number of computers at home and times the children spent on holiday in the past 12 months. The FAS was collected in seven categories (from 0 the lowest to 7 the highest) and was re-coded into three groups for the analysis, i.e., low (0 to 3 ), intermediate (4 to 5) and high family income (6 to 7). The psychometric properties of the FAS are acceptable and support its use as a self-reported adolescents' measure [45].

In order to assess dimensions of children's physical, psychological and social health-related quality of life (HRQoL) that could be associated with emotional and behavioural problems, the KIDSCREEN-52 was used [46]. It is a generic self-reported questionnaire for children and adolescents from 8 to 18 years with good psychometric properties [46]. It is intended to assess HRQoL from the child's/adolescent's perspective and focus on physical, mental and social dimensions of wellbeing. The KIDSCREEN-52 instrument aims at identifying children and adolescents at risk with regard to their subjective health. It includes ten HRQoL dimensions: 1) physical wellbeing; 2) psychological wellbeing; 3) moods and emotions; 4) self-perception; 5) autonomy; 6) parent relations and home life; 7) social support and peers; 8) school environment; 9) social acceptance and bullying; and 10) financial resources. The KIDSCREEN52 questionnaire assesses either the frequency of behaviour/feelings or, in fewer cases, the intensity of an attitude. Both possible item formats use a 5-point Likert response scale, and the recall period is 1 week. Total score from each dimension is ranging from 0 to 100, with higher scores indicating higher HRQoL. The Greek version of the instrument has been found to have good reliability [47]. Convergent and discriminatory validity, tested against information about the adolescents' physical and mental health have also been found at satisfactory levels [46]. The KIDSCREEN-52 versions for adolescents and parents were used in the present study.

\subsection{Statistical Analysis}

Factor analyses of the Greek version of the SDQ were conducted using Varimax Orthogonal Transformation with number of factors forced to be five. The internal consistency of different SDQ scales was analyzed with Cronbach's $\alpha$. Alpha coefficient of 0.7 or higher were considered acceptable. The inter-rater agreement between parent and self-report scores was analyzed with Pearson's correlation coefficient. Correlation coefficient between 0.1 and 0.3 were considered low, between 0.31 and 0.5 moderate and those over 0.5 were considered high. Intra-class correlation coefficients (ICCs) were computed in order to assess test-retest stability. A coefficient of 0.6 or higher was considered as evidence for good test-retest stability.

Construct-related validity was evaluated based on previously developed hypotheses regarding family socioeconomic status. It was expected that adolescents coming from disadvantaged socioeconomic background would also report more emotional and behavioral problems. Mean T-values of SDQ scales were computed according to socioeconomic status. Construct validity was assessed by calculating Cohen's effect sizes (ES, $d$ ). Effect sizes of $0.2-0.5$ were considered small, between $0.51-0.81$ moderate and over 0.8 were considered large. Pearson correlation coefficients were computed to analyze convergent validity between SDQ scale scores and the 10 dimensions of the KIDSCREEN-52 questionnaire. Convergent validity was considered to be demonstrated when correlations between theoretically comparable dimensions were significantly higher than correlations between theoretically different dimensions.

\section{RESULTS}

Table 1 shows the factor loadings of each item after five-factor solution was forced for parent and self-reported SDQ. For both parent and self-reports, most items loaded onto their predicted factors indicating that the factors produced on the basis of the Greek sample were consistent with the original scales of the SDQ. For self-reports, the first five factors had eigenvalues $>1.0$, i.e., 4.4, 2.6, 1.5, 1.3, 1.2, and accounted for $44.1 \%$ of the total variance. The item "Other people my age generally like $m e$ " that was supposed to load on peer problems factor loaded on prosocial behaviour factor, while the item "I usually do as I am told" loaded on the emotional symptoms factor instead on the conduct problems factor. Regarding parent-reports, the first five factors had eigenvalues $>1.0$, i.e., 4.5, 2.6, 1.7, 1.3, 1.2, and accounted for $44.4 \%$ of the total variance. Discrepancies from the original scales of the SDQ were identified in relation to two items. More specifically, the item "Steals from home, school or elsewhere/I take things that are not mine" loaded on the peer problems instead for the conduct problems factor and the item "Gets on better with adults than with other people/I get on better with adults" loaded 
Table 1. Factor loadings of SDQ items in parent (left columns) and self-reports (right columns).

\begin{tabular}{|c|c|c|c|c|c|c|c|c|c|c|}
\hline \multirow{3}{*}{$\frac{\text { SDQ items }}{\text { Nice to other people }}$} & \multicolumn{10}{|c|}{ Hypothesized factors } \\
\hline & \multicolumn{2}{|c|}{ Prosocial behaviour } & \multicolumn{2}{|c|}{ Hyperactivity/inattention } & \multicolumn{2}{|c|}{ Emotional symptoms } & \multicolumn{2}{|c|}{ Conduct problems } & \multicolumn{2}{|c|}{ Peer problems } \\
\hline & 0.59 & 0.64 & & & & & & & & \\
\hline Shares with others & 0.59 & 0.61 & & & & & & & & \\
\hline Helpful if someone hurt & 0.69 & 0.68 & & & & & & & & \\
\hline Kind to younger children & 0.57 & 0.57 & & & & & & & & \\
\hline Volunteers to help & 0.68 & 0.67 & & & & & & & & \\
\hline Restless & & & 0.83 & 0.71 & & & & & & \\
\hline Constantly "fidgeting" & & & 0.76 & 0.75 & & & & & & \\
\hline Has difficulty concentrating & & & 0.59 & 0.44 & & & & & & \\
\hline Thinks before doing things ${ }^{*}$ & & & 0.64 & 0.44 & & & & & & \\
\hline Has good attention ${ }^{*}$ & & & 0.70 & 0.75 & & & & & & \\
\hline Has headaches and stomach aches & & & & & 0.53 & 0.43 & & & & \\
\hline Worries a lot & & & & & 0.58 & 0.57 & & & & \\
\hline Often unhappy & & & & & 0.68 & 0.61 & & & & \\
\hline Nervous & & & & & 0.56 & 0.44 & & & & \\
\hline Has fears & & & & & 0.51 & 0.43 & & & & \\
\hline Loses temper & & & & & & & 0.43 & 0.60 & & \\
\hline Does as told* & & & & & & -0.46 & -0.41 & 0.23 & & \\
\hline Fights a lot & & & & & & & 0.42 & 0.52 & & \\
\hline Accused of lying & & & & & & & 0.49 & 0.65 & & \\
\hline Steals from home and school & & & & & & & -0.20 & 0.73 & 0.64 & \\
\hline Usually alone & & & & & & & & & 0.56 & 0.62 \\
\hline Has good friend(s) ${ }^{*}$ & & & & & & & & & -0.47 & -0.47 \\
\hline Popular $^{*}$ & & 0.49 & & & & & & & 0.43 & -0.13 \\
\hline "Picked on" or bullied & & & & & & & & & 0.69 & 0.49 \\
\hline Prefers adults & & & & & 0.44 & & & & 0.13 & 0.47 \\
\hline
\end{tabular}

*Reversely coded.

on the emotional symptoms rather than the peer problems factor.

The internal consistency coefficient (Cronbach's $\alpha$ ) for the total difficulties score, which includes all scales except for the prosocial behaviour, was 0.78 for parentreported SDQ and 0.77 for self-reported SDQ (Table 2). The Cronbach's $\alpha$ for conduct problems and peer problems were low in both parent and self-reports.

Table 2 presents correlations of parent and self-reported SDQ scales. Prosocial behaviour scale was negatively correlated with other scales as expected. For parent-reports, each scale was correlated significantly with the other, with correlations ranging from -0.22 (prosocial behaviour and conduct problems scales) to 0.45 (hyperactivity/inattention and emotional symptoms scales). The correlations for self-reported SDQ scales were all significant ranging from 0.19 (hyperactivity/ inattention and peer problems scales) to 0.47 (hyperactivity/inattention and conduct problems scales). The correlations of all parent and self-reported SDQ scales with the total difficulties score were significant as well.

In Table $\mathbf{2}$ are presented correlations between parent and self-reported SDQ scales. All scales were correlated significantly, with correlations ranging from 0.33 (con- 
Table 2. Cross-scale correlations and Cronbach's $\alpha$ of SDQ parent and self-reports, test-retest and inter-rater correlations.

\begin{tabular}{|c|c|c|c|c|c|c|c|}
\hline & $\alpha$ & $\begin{array}{c}\text { Total } \\
\text { difficulties } \\
\end{array}$ & $\begin{array}{l}\text { Emotional } \\
\text { symptoms }\end{array}$ & $\begin{array}{c}\text { Conduct } \\
\text { problems }\end{array}$ & Hyperactivity/inattention & Peer problems & Test-retest correlations \\
\hline \multicolumn{8}{|l|}{ Self-reports } \\
\hline Total difficulties & 0.77 & & & & & & 0.89 \\
\hline Emotional symptoms & 0.73 & $0.78^{*}$ & & & & & 0.83 \\
\hline Conduct problems & 0.56 & $0.63^{*}$ & $0.26^{*}$ & & & & 0.85 \\
\hline Hyperactivity/inattention & 0.63 & $0.79^{*}$ & $0.46^{*}$ & $0.41^{*}$ & & & 0.76 \\
\hline Peer problems & 0.50 & $0.65^{*}$ & $0.41^{*}$ & $0.26^{*}$ & $0.27^{*}$ & & 0.81 \\
\hline Prosocial behaviour & 0.72 & $-0.22^{*}$ & $-0.34^{*}$ & $-0.22^{*}$ & $-0.27^{*}$ & $-0.26^{*}$ & 0.65 \\
\hline \multicolumn{8}{|l|}{ Parent-reports } \\
\hline Total difficulties & 0.78 & & & & & & 0.84 \\
\hline Emotional symptoms & 0.71 & $0.78^{*}$ & & & & & 0.82 \\
\hline Conduct problems & 0.57 & $0.71^{*}$ & $0.38^{*}$ & & & & 0.88 \\
\hline Hyperactivity/inattention & 0.70 & $0.78^{*}$ & $0.44^{*}$ & $0.47^{*}$ & & & 0.77 \\
\hline Peer problems & 0.53 & $0.57^{*}$ & $0.35^{*}$ & $0.23^{*}$ & $0.19^{*}$ & & 0.89 \\
\hline Prosocial behaviour & 0.72 & $-0.29^{*}$ & $-0.27^{* *}$ & $-0.30^{*}$ & $-0.21^{*}$ & $-0.27^{*}$ & 0.76 \\
\hline \multicolumn{8}{|l|}{ Inter-rater correlations } \\
\hline Total difficulties & $0.45^{*}$ & & & & & & \\
\hline Emotional symptoms & $0.41^{*}$ & & & & & & \\
\hline Conduct problems & $0.33^{*}$ & & & & & & \\
\hline Hyperactivity/inattention & $0.41^{*}$ & & & & & & \\
\hline Peer problems & $0.35^{*}$ & & & & & & \\
\hline Prosocial behaviour & $0.36^{*}$ & & & & & & \\
\hline
\end{tabular}

${ }^{*} P<0.001 ;{ }^{* *} P<0.05$.

duct problems scale) to 0.45 (total difficulties scale). Correlations did not vary according to different age groups or gender. Test-retest stability analysis showed ICCs above 0.60 in all scales for both parent and selfreports (Table 2 ).

Table 3 shows means and standard deviations obtained for scale scores of the parent and self-reported SDQ for boys and girls according to different age groups. Results showed that parents reported significantly higher scores for girls compared to boys with respect to total difficulties, emotional symptoms and prosocial behaviour scales $(P<0.001)$.

In relation to age, parents reported significant higher scores for adolescents aged 15 to 17 years than those aged 11 to 14 years on total difficulties $(P<0.05)$ and emotional symptoms scales $(P<0.001)$. No significant interaction was found between sex and age.

As far as self-reports are concerned, girls reported higher mean scores on emotional symptoms $(P<0.001)$ and prosocial behaviour scales $(P=0.002)$. Age differences revealed that adolescents aged 15 to 17 years compared to those aged 11 to 14 years, reported significantly higher mean scores on all scales except for prosocial behaviour scale, namely, total difficulties $(P<0.001)$, emotional symptoms $(P<0.001)$, conduct problems $(P=$ $0.007)$, hyperactivity/inattention $(P<0.001)$, and peer problems scales $(P=0.044)$. The score on the prosocial behaviour scale was significantly lower $(P=0.034)$ in adolescents aged 15 to 17 years with mean (SD) equal to $7.9 \pm 1.9$ than those aged 11 to 14 years with mean (SD) equal to $8.2 \pm 1.8$. A significant sex $\times$ age interaction was found for the emotional symptoms scale $(P=0.034)$ proclaiming that females had higher scores on the emotional symptoms scale, with the difference being more significant for those aged 15 to 17 years than those aged 11 to 14 years.

Univariate analysis showed statistically significant differences in SDQ scores between adolescents reporting 
Table 3. Scale scores (mean \pm SD) of SDQ parent and self-reports across sex and age groups.

\begin{tabular}{cccccc}
\hline & & \multicolumn{2}{c}{$11-14$ years } & & \multicolumn{2}{c}{$15-17$ years } \\
\hline & Total & Boys & Girls & Boys & \\
\hline Parent-reports & & & & & \\
Prosocial behaviour & $8.5 \pm 1.7$ & $8.5 \pm 1.5$ & $8.6 \pm 1.5$ & $8.0 \pm 2.1$ & $8.6 \pm 1.7$ \\
Hyperactivity/inattention & $2.9 \pm 2.2$ & $3.0 \pm 2.3$ & $2.6 \pm 2.1$ & $3.3 \pm 2.2$ & $2.9 \pm 2.2$ \\
Emotional symptoms & $2.3 \pm 1.9$ & $1.7 \pm 1.8$ & $2.3 \pm 1.8$ & $1.9 \pm 1.9$ & $2.8 \pm 2.1$ \\
Conduct problems & $2.0 \pm 1.5$ & $2.0 \pm 1.6$ & $2.0 \pm 1.6$ & $2.1 \pm 1.6$ & $2.0 \pm 1.5$ \\
Peer problems & $1.6 \pm 1.5$ & $1.4 \pm 1.5$ & $1.5 \pm 1.4$ & $1.8 \pm 1.6$ & $1.6 \pm 1.4$ \\
Total difficulties & $8.8 \pm 5.1$ & $8.3 \pm 5.1$ & $8.6 \pm 5.1$ & $9.0 \pm 5.3$ & $9.2 \pm 5.2$ \\
Self-reports & & & & & $8.2 \pm 1.8$ \\
Prosocial behaviour & $8.1 \pm 1.8$ & $7.9 \pm 2.0$ & $8.4 \pm 1.6$ & $7.5 \pm 2.1$ & $4.1 \pm 2.2$ \\
Hyperactivity/inattention & $3.6 \pm 2.2$ & $3.1 \pm 2.2$ & $3.1 \pm 2.1$ & $3.9 \pm 2.2$ & $3.7 \pm 2.1$ \\
Emotional symptoms & $3.0 \pm 2.1$ & $2.3 \pm 2.2$ & $3.1 \pm 2.0$ & $2.5 \pm 2.0$ & $3.1 \pm 1.5$ \\
Conduct problems & $2.0 \pm 1.5$ & $3.0 \pm 1.7$ & $2.8 \pm 1.4$ & $3.0 \pm 1.5$ & $2.0 \pm 1.6$ \\
Peer problems & $1.8 \pm 1.7$ & $1.9 \pm 2.0$ & $1.7 \pm 1.6$ & $1.9 \pm 1.8$ & $11.3 \pm 5.4$ \\
Total difficulties & $11.3 \pm 5.2$ & $10.2 \pm 6.5$ & $10.5 \pm 5.0$ & & $12.9 \pm 4.9$ \\
\hline
\end{tabular}

low versus high family income (Table 4) except for conduct problems and prosocial behaviour scores. No significant differences were obtained between adolescents of high versus medium family income. Medium versus low family income differentiated significantly adolescents in scale scores for emotional symptoms, peer problems and total difficulties scores. However, only small ES $(d)$ were found for all SDQ scale mean $(d>0.5)$. The largest effect size was observed for emotional symptoms scale.

Convergent validity analysis showed moderate to high-level correlations for the expected relationships (Table 5). The SDQ emotional symptoms scale, as well as the total difficulties score were correlated highest with the KIDSCREEN-52 moods and emotions dimension. Moderate correlations of the SDQ emotional scale were also observed with the KIDSCREEN-52 dimensions of self-perception, psychological well-being, parent relation, physical well-being and social support and peers. The SDQ hyperactivity/inattention scale was correlated highest with the KIDSCREEN-52 dimensions of school environment, moods and emotions and parent relations dimensions. The SDQ peer problems scale was correlated highest with the KIDSCREEN-52 dimensions of peers and social support, moods and emotions and social acceptance and bullying.

\section{DISCUSSION}

The present study aimed at investigating the psycho- metric properties of the Greek version of the SDQ in a school-based sample of adolescents and their parents. The main results encourage the use of this widely used questionnaire for Greek adolescents and are catalogued as follows. Factor analysis of the SDQ yielded five factors, in consistency with the proposed structure of the original questionnaire and the hypothesised scales of emotional symptoms, conduct problems, hyperactivity/ inattention, peer problems, and prosocial behaviour. The internal consistency of SDQ scales was acceptable. Furthermore, the abovementioned psychometric properties were highly similar for parent and self-report versions. Finally, the parent-adolescent agreement for SDQ scores was reasonable.

The reliability of the SDQ scales also appeared to be reasonable. However, the internal consistency of two scales, viz. conduct problems and peer problems, in both parent and self-reports, was below acceptable limits. Given that SDQ scales except for total difficulties scale consist of only 5 items, this result was not surprising.

While the original five-factor structure of the SDQ was generally confirmed, two items in the conduct problems scale, viz., "I take things that are not mine" and "I usually do as I am told", loaded more strongly onto the peer problems scale. Studies outside the UK have also noted unexpected factor loadings for the item "I usually do as I am told" $[7,21,33,35]$. The findings from the present study can therefore be seen as adding to existing evidence, questioning the utility of this item as an indi- 
Table 4. Correlations of family income with SDQ self-reports.

\begin{tabular}{cccccccc}
\hline \multicolumn{1}{c}{ Low } & \multicolumn{3}{c}{ Family income } \\
\hline & \multicolumn{3}{c}{ Medium } & \multicolumn{3}{c}{ High } \\
\hline Self-reports & Mean T-value & (SD) & Mean T-value & (SD) & Mean T-value & (SD) & Effect size (low vs. high) \\
\hline Emotional symptoms & 51.82 & $(10.04)$ & 49.28 & $(9.76)$ & 48.60 & $(9.94)$ & 0.32 \\
Conduct problems & 50.49 & $(10.02)$ & 49.55 & $(10.28)$ & 50.39 & $(9.35)$ & 0.01 \\
Hyperactivity/inattention & 51.20 & $(9.35)$ & 50.08 & $(9.78)$ & 49.21 & $(10.43)$ & 0.20 \\
Peer problems & 51.69 & $(10.60)$ & 48.91 & $(9.57)$ & 48.77 & $(9.14)$ & 0.29 \\
Prosocial behaviour & 50.61 & $(9.40)$ & 49.36 & $(10.39)$ & 49.63 & $(10.77)$ & 0.10 \\
Total difficulties & 51.67 & $(9.86)$ & 49.00 & $(10.07)$ & 49.49 & $(9.62)$ & 0.22
\end{tabular}

$P<0.05$ for mean differences (low vs. high) except for conduct problems and prosocial behaviour scales. Effect size $(d): 0.20=$ small, $0.50=$ moderate, $0.80=$ large.

Table 5. Correlations of KIDSCREEN-52 dimensions with scales of SDQ self-reports.

\begin{tabular}{ccccccc}
\hline & \multicolumn{3}{c}{ SDQ scales } \\
\hline KIDSCREEN-52 Dimensions & $\begin{array}{c}\text { Emotional } \\
\text { symptoms }\end{array}$ & $\begin{array}{c}\text { Conduct } \\
\text { problems }\end{array}$ & $\begin{array}{c}\text { Hyperactivity/ } \\
\text { inattention }\end{array}$ & $\begin{array}{c}\text { Peer } \\
\text { problems }\end{array}$ & $\begin{array}{c}\text { Prosocial } \\
\text { behaviour }\end{array}$ & $\begin{array}{c}\text { Total } \\
\text { difficulties }\end{array}$ \\
\hline Physical well-being & -0.33 & -0.12 & -0.23 & -0.19 & 0.16 & -0.31 \\
Psychological well-being & -0.40 & -0.11 & -0.25 & -0.27 & 0.16 & -0.38 \\
Moods \& emotions & -0.58 & -0.23 & -0.38 & -0.35 & 0.17 & -0.56 \\
Self-perception & -0.47 & -0.18 & -0.30 & -0.25 & 0.17 & -0.44 \\
Autonomy & -0.28 & -0.12 & -0.12 & -0.17 & 0.13 & -0.22 \\
Parent relation \& home life & -0.34 & -0.23 & -0.31 & -0.23 & 0.19 & -0.38 \\
Peers \& social support & -0.31 & -0.16 & -0.12 & -0.40 & 0.18 & -0.30 \\
School environment & -0.22 & -0.21 & -0.41 & -0.18 & 0.29 & -0.37 \\
Social acceptance and bullying & -0.26 & -0.11 & -0.16 & -0.34 & 0.19 & -0.29 \\
Financial resources & -0.26 & -0.20 & -0.20 & -0.25 & 0.11 & -0.26 \\
\hline
\end{tabular}

All correlations were significant at $P<0.001$. Correlation coefficients: $0.1-0.3=$ low, $0.31-0.5=$ moderate, $>0.5=$ high.

cator of conduct problems in children and adolescents in different cultural settings.

Sex and age effects on the Greek SDQ scores did not agree well with those observed by comparable studies in other countries [6]. Greek parents reported higher levels of hyperactivity/inattention and conduct problems for girls than boys. Also, unlike developmental changes in clinical prevalence rates as detected by epidemiological studies, the hyperactivity/inattention score increased with increasing age. The different factor loadings and specific educational circumstances in Greece, i.e., secondary school adolescents face extremely competitive and stressful university entry exams, may be involved and should be further explored in future research.

The present analysis showed statistically significant differences in SDQ scores between adolescents reporting low versus high family income, except for conduct problems and prosocial behaviour. Medium versus low family income differentiated significantly adolescents in scale scores for emotional symptoms, peer problems, and total difficulties. However, no significant differences were observed between adolescents of high versus medium family income. Previous research has confirmed the above mentioned finding, suggesting that children and adolescent with lower socioeconomic status score significantly higher on the hyperactivity/inattention and peer problems scales [23,36].

With respect to the relationships between the mainly psychologically oriented SDQ scales and the generic HRQOL dimensions of the KIDSCREEN-52, it can be claimed that correlations between the two instruments were as predicted. The most significant correlations emer- 
ged in general between scales and dimensions tapping similar aspects of emotional and behavioural problems, e.g., SDQ peer problems with KIDSCREEN-52 peers and social support, SDQ hyperactivity/inattention with KIDSCREEN-52 school environment, SDQ emotional symptoms with KIDSCREEN-52 moods and emotions, self-perception and psychological wellbeing. Also, it should be noted that correlations were satisfactory, given the substantial difference in length and item contents of the two measures.

It should be acknowledged that the present study has a number of limitations. Firstly, the study relied exclusively on participants from the general population. As a result, the psychometric properties of the SDQ in clinically referred Greek adolescents remain to be established. Secondly, only SDQ parent and self-reports were obtained, without including teacher reports. Teachers are valuable informants, as it is generally acknowledged that they can provide important additional information on the strengths and difficulties of children and adolescents. Thirdly, SDQ scales were not validated against other questionnaires detecting psychopathology. Finally, comparison of SDQ scores with psychiatric diagnoses measured through standardised interviews would certainly have strengthened this study.

Despite these limitations, the present results are encouraging by providing evidence of the psychometric qualities of the SDQ in Greece. Findings are generally consistent with numerous studies elsewhere. However, future studies with teacher reports are needed. Future studies are also needed to validate the Greek version of the SDQ compared with other questionnaires of psychopathology and psychiatric diagnoses.

\section{ACKNOWLEDGEMENTS}

The KIDSCREEN project was financed by a grant from the European Commission (QLG-CT-2000-00751) within the EC 5th Framework-Programme "Quality of Life and Management of Living Resources".

\section{REFERENCES}

[1] World Health Organization (2005) Child and adolescent mental health policies and plans. World Health Organization, Geneva.

[2] Patel, V., Kieling, C., Maulik, P.K. and Divan, G. (2013) Improving access to care for children with mental disorders: A global perspective. Archives of Disease in Childhood, 98, 323-327.

http://dx.doi.org/10.1136/archdischild-2012-302079

[3] Kessler, R.C. (2000) Psychiatric epidemiology: Selected recent advances and future directions. Bulletin of the World Health Organization, 78, 464-474.

[4] Goodman, R. (1997) The strengths and difficulties ques- tionnaire: A research note. Journal of Child Psychology and Psychiatry, 38, 581-586.

http://dx.doi.org/10.1111/j.1469-7610.1997.tb01545.x

[5] Vostanis, P. (2006) Strengths and difficulties questionnaire: Research and clinical applications. Current Opinion in Psychiatry, 19, 367-372.

http://dx.doi.org/10.1097/01.yco.0000228755.72366.05

[6] Stone, L.L., Otten, R., Engels, R.C.M.E., Vermulst, A.A. and Janssens, J.M.A.M. (2010) Psychometric properties of the parent and teacher versions of the strengths and difficulties questionnaire for 4- to 12-year-olds: A review. Clinical Child and Family Psychology Review, 13, 254 274. http://dx.doi.org/10.1007/s10567-010-0071-2

[7] Goodman, R. (2001) Psychometric properties of the strengths and difficulties questionnaire. Journal of the American Academy of Child and Adolescent Psychiatry, 40, 1337-1345.

http://dx.doi.org/10.1097/00004583-200111000-00015

[8] He, J.-P., Burstein, M., Schmitz, A. and Merikangas, K.R. (2013) The Strengths and Difficulties Questionnaire (SDQ): The factor structure and scale validation in U.S. adolescents. Journal of Abnormal Child Psychology, 41, 583595. http://dx.doi.org/10.1007/s10802-012-9696-6

[9] Gómez-Beneyto, M., Nolasco, A., Moncho, J., PereyraZamora, P., Tamayo-Fonseca, N., Munarriz, M., Salazar, J., Tabarés-Seisdedos, R. and Girón, M. (2013) Psychometric behaviour of the strengths and difficulties questionnaire (SDQ) in the Spanish national health survey 2006. BMC Psychiatry, 13, 95.

http://dx.doi.org/10.1186/1471-244X-13-95

[10] Niclasen, J., Skovgaard, A.M., Andersen, A.-M.N., Sømhovd, M.J. and Obel, C. (2013) A confirmatory approach to examining the factor structure of the Strengths and Difficulties Questionnaire (SDQ): A large scale cohort study. Journal of Abnormal Child Psychology, 41, 355365. http://dx.doi.org/10.1007/s10802-012-9683-y

[11] Ruchkin, V., Koposov, R., Vermeiren, R. and SchwabStone, M. (2012) The Strength and Difficulties Questionnaire: Russian validation of the teacher version and comparison of teacher and student reports. Journal of Adolescence, 35, 87-96.

http://dx.doi.org/10.1016/j.adolescence.2011.06.003

[12] Van de Looij-Jansen, P.M., Goedhart, A.W., De Wilde, E.J. and Treffers, P.D.A. (2011) Confirmatory factor analysis and factorial invariance analysis of the adolescent self-report Strengths and Difficulties Questionnaire: How important are method effects and minor factors? British Journal of Clinical Psychology, 50, 127-144. http://dx.doi.org/10.1348/014466510X498174

[13] Richter, J., Sagatun, A., Heyerdahl, S., Oppedal, B. and Røysamb, E. (2011) The Strengths and Difficulties Questionnaire (SDQ) - Self-report. An analysis of its structure in a multiethnic urban adolescent sample. Journal of Child Psychology and Psychiatry, 52, 1002-1011. http://dx.doi.org/10.1111/j.1469-7610.2011.02372.x

[14] Giannakopoulos, G., Tzavara, C., Dimitrakaki, C., Kolaitis, G., Rotsika, V. and Tountas, Y. (2009) The factor structure of the Strengths and Difficulties Questionnaire (SDQ) in Greek adolescents. Annals of General Psy- 
chiatry, 8, 20. http://dx.doi.org/10.1186/1744-859X-8-20

[15] Yao, S., Zhang, C., Zhu, X., Jing, X., McWhinnie, C.M. and Abela, J.R.Z. (2009) Measuring adolescent psychopathology: Psychometric properties of the self-report Strengths and Difficulties Questionnaire in a sample of Chinese adolescents. Journal of Adolescent Health, 45, 55-62. http://dx.doi.org/10.1016/j.jadohealth.2008.11.006

[16] Rothenberger, A., Becker, A., Erhart, M., Wille, N., Ravens-Sieberer, U. and Bella Study Group (2008) Psychometric properties of the parent Strengths and Difficulties Questionnaire in the general population of German children and adolescents: Results of the BELLA study. European Child and Adolescent Psychiatry, 17, 99-105. http://dx.doi.org/10.1007/s00787-008-1011-2

[17] Van Roy, B., Veenstra, M. and Clench-Aas, J. (2008) Construct validity of the five-factor Strengths and Difficulties Questionnaire (SDQ) in pre-, early, and late adolescence. Journal of Child Psychology and Psychiatry, 49, 1304-1312. http://dx.doi.org/10.1111/j.1469-7610.2008.01942.x

[18] Lundh, L.-G., Wångby-Lundh, M. and Bjärehed, J. (2008) Self-reported emotional and behavioral problems in Swedish 14 to 15-year-old adolescents: A study with the self-report version of the Strengths and Difficulties Questionnaire. Scandinavian Journal of Psychology, 49, 523532. http://dx.doi.org/10.1111/j.1467-9450.2008.00668.x

[19] Matsuishi, T., Nagano, M., Araki, Y., Tanaka, Y., Iwasaki, M., Yamashita, Y., Nagamitsu, S., Iizuka, C., Ohya, T., Shibuya, K., Hara, M., Matsuda, K., Tsuda, A. and Kakuma, T. (2008) Scale properties of the Japanese version of the Strengths and Difficulties Questionnaire (SDQ): A study of infant and school children in community samples. Brain and Development, 30, 410-415. http://dx.doi.org/10.1016/j.braindev.2007.12.003

[20] Hayes, L. (2007) Problem behaviours in early primary school children: Australian normative data using the Strengths and Difficulties Questionnaire. Australian and New Zealand Journal of Psychiatry, 41, 231-238. http://dx.doi.org/10.1080/00048670601172715

[21] Hawes, D.J. and Dadds, M.R. (2004) Australian data and psychometric properties of the Strengths and Difficulties Questionnaire. Australian and New Zealand Journal of Psychiatry, 38, 644-651.

http://dx.doi.org/10.1080/j.1440-1614.2004.01427.x

[22] Becker, A., Woerner, W., Hasselhorn, M., Banaschewski, T. and Rothenberger, A. (2004) Validation of the parent and teacher SDQ in a clinical sample. European Child and Adolescent Psychiatry, 13, ii11-ii16. http://dx.doi.org/10.1007/s00787-004-2003-5

[23] Woerner, W., Becker, A. and Rothenberger, A. (2004) Normative data and scale properties of the German parent SDQ. European Child and Adolescent Psychiatry, 13, ii3-ii10. http://dx.doi.org/10.1007/s00787-004-2002-6

[24] Koskelainen, M., Sourander, A. and Vauras, M. (2001) Self-reported strengths and difficulties in a community sample of Finnish adolescents. European Child and Adolescent Psychiatry, 10, 180-185. http://dx.doi.org/10.1007/s007870170024

[25] Liu, S.-K., Chien, Y.-L., Shang, C.-Y., Lin, C.-H., Liu,
Y.-C. and Gau, S.S.-F. (2013) Psychometric properties of the Chinese version of Strength and Difficulties Questionnaire. Comprehensive Psychiatry, 54, 720-730. http://dx.doi.org/10.1016/j.comppsych.2013.01.002

[26] Borg, A.-M., Kaukonen, P., Salmelin, R., Joukamaa, M. and Tamminen, T. (2012) Reliability of the Strengths and Difficulties Questionnaire among Finnish 4-9-year-old children. Nordic Journal of Psychiatry, 66, 403-413. http://dx.doi.org/10.3109/08039488.2012.660706

[27] Mansbach-Kleinfeld, I., Apter, A., Farbstein, I., Levine, S.Z. and Ponizovsky, A.M. (2010) A population-based psychometric validation study of the Strengths and Difficulties Questionnaire-Hebrew version. Frontiers in Psychiatry, 1, 151. http://dx.doi.org/10.3389/fpsyt.2010.00151

[28] Lai, K.Y.C., Luk, E.S.L., Leung, P.W.L., Wong, A.S.Y., Law, L. and Ho, K. (2010) Validation of the Chinese version of the Strengths and Difficulties Questionnaire in Hong Kong. Social Psychiatry and Psychiatric Epidemiology, 45, 1179-1186.

http://dx.doi.org/10.1007/s00127-009-0152-z

[29] Shahrivar, Z., Tehrani-Doost, M., Pakbaz, B., Rezaie, A. and Ahmadi, F. (2009) Normative data and psychometric properties of the parent and teacher versions of the Strengths and Difficulties Questionnaire (SDQ) in an Iranian community sample. Journal of Research in $\mathrm{Me}$ dical Science, 14, 69-77.

[30] Shojaei, T., Wazana, A., Pitrou, I. and Kovess, V. (2009) The Strengths and Difficulties Questionnaire: Validation study in French school-aged children and cross-cultural comparisons. Social Psychiatry and Psychiatric Epidemiology, 44, 740-747.

http://dx.doi.org/10.1007/s00127-008-0489-8

[31] Rønning, J.A., Handegaard, B.H., Sourander, A. and Mørch, W.-T. (2004) The Strengths and Difficulties SelfReport Questionnaire as a screening instrument in Norwegian community samples. European Child and Adolescent Psychiatry, 13, 73-82. http://dx.doi.org/10.1007/s00787-004-0356-4

[32] Van Widenfelt, B.M., Goedhart, A.W., Treffers, P.D.A. and Goodman, R. (2003) Dutch version of the Strengths and Difficulties Questionnaire (SDQ). European Child and Adolescent Psychiatry, 12, 281-289. http://dx.doi.org/10.1007/s00787-003-0341-3

[33] Muris, P., Meesters, C. and van den Berg, F. (2003) The Strengths and Difficulties Questionnaire (SDQ) - Further evidence for its reliability and validity in a community sample of Dutch children and adolescents. European Child and Adolescent Psychiatry, 12, 1-8. http://dx.doi.org/10.1007/s00787-003-0298-2

[34] Mellor, D. (2004) Furthering the use of the Strengths and Difficulties Questionnaire: Reliability with younger child respondents. Psychological Assessment, 16, 396-401. http://dx.doi.org/10.1037/1040-3590.16.4.396

[35] Smedje, H., Broman, J.E., Hetta, J. and von Knorring, A.L. (1999) Psychometric properties of a Swedish version of the "Strengths and Difficulties Questionnaire". European Child and Adolescent Psychiatry, 8, 63-70. http://dx.doi.org/10.1007/s007870050086 
[36] Marzocchi, G.M., Capron, C., Di Pietro, M., Duran Tauleria, E., Duyme, M., Frigerio, A., Gaspar, M.F., Hamilton, H., Pithon, G., Simoes, A. and Therond, C. (2004) The use of the Strengths and Difficulties Questionnaire (SDQ) in Southern European countries. European Child and Adolescent Psychiatry, 13, ii40-ii46. http://dx.doi.org/10.1007/s00787-004-2007-1

[37] Ruchkin, V., Jones, S., Vermeiren, R. and Schwab-Stone, M. (2008) The Strengths and Difficulties Questionnaire: The self-report version in American urban and suburban youth. Psychological Assessment, 20, 175-182. http://dx.doi.org/10.1037/1040-3590.20.2.175

[38] Du, Y., Kou, J. and Coghill, D. (2008) The validity, reliability and normative scores of the parent, teacher and self report versions of the Strengths and Difficulties Questionnaire in China. Child and Adolescent Psychiatry and Mental Health, 2, 8. http://dx.doi.org/10.1186/1753-2000-2-8

[39] Mieloo, C.L., Bevaart, F., Donker, M.C.H., van Oort, F.V.A., Raat, H. and Jansen, W. (2013) Validation of the SDQ in a multi-ethnic population of young children. European Journal of Public Health. http://dx.doi.org/10.1093/eurpub/ckt100

[40] Koskelainen, M., Sourander, A. and Kaljonen, A. (2000) The Strengths and Difficulties Questionnaire among Finnish school-aged children and adolescents. European Child and Adolescent Psychiatry, 9, 277-284. http://dx.doi.org/10.1007/s007870070031

[41] Goodman, R., Meltzer, H. and Bailey, V. (1998) The Strengths and Difficulties Questionnaire: A pilot study on the validity of the self-report version. European Child and Adolescent Psychiatry, 7, 125-130. http://dx.doi.org/10.1007/s007870050057

[42] Rajmil, L., Berra, S., von Rueden, U., Tebe, C., Erhart,
M., Gosch, A., Alonso, J., Bisegger, C. and RavensSieberer, U. (2004) Representativity of 12 national surveys of children and adolescents 8 - 18 years old included in the KIDSCREEN HRQoL study. Quality of Life Research, 13, 1576.

[43] Bullinger, M., Anderson, R., Cella, D. and Aaronson, N. (1995) Developing and evaluating cross-cultural instruments from minimum requirements to optimal models. In: Shumaker, S.A. and Berzon, R.A., Eds., The International Assessment of Health-Related Quality of Life, Rapid Communications, New York, 83-91.

[44] Currie, C.E., Elton, R.A., Todd, J. and Platt, S. (1997) Indicators of socioeconomic status for adolescents: The WHO health behaviour in school-aged children survey. Health Education Research, 12, 385-397. http://dx.doi.org/10.1093/her/12.3.385

[45] Boyce, W., Torsheim, T., Currie, C. and Zambon, A. (2006) The family affluence scale as a measure of national wealth: Validation of an adolescent self-reported measure. Social Indicators Research, 78, 473-487. http://dx.doi.org/10.1007/s11205-005-1607-6

[46] Ravens-Sieberer, U., Gosch, A., Rajmil, L., Erhart, M., Bruil, J., Duer, W., Auquier, P., Power, M., Abel, T., Czemy, L., Mazur, J., Czimbalmos, A., Tountas, Y., Hagquist, C., Kilroe, J. and Group, T.E.K. (2005) KIDSCREEN-52 quality-of-life measure for children and adolescents. Expert Review of Pharmacoeconomics Outcomes Research, 5, 353-364. http://dx.doi.org/10.1586/14737167.5.3.353

[47] Tzavara, C., Tzonou, A., Zervas, I., Ravens-Sieberer, U., Dimitrakaki, C. and Tountas, Y. (2012) Reliability and validity of the KIDSCREEN-52 health-related quality of life questionnaire in a Greek adolescent population. $A n$ nals of General Psychiatry, 11, 3. http://dx.doi.org/10.1186/1744-859X-11-3 